\title{
Assessment of the Parameters of a Shock Wave on the Wall of an Explosion Cavity with the Refraction of a Detonation Wave of Emulsion Explosives
}

\author{
Pavel Igorevich Afanasev ${ }^{1, *(\mathbb{D})}$ and Khairullo Faizullaevich Makhmudov ${ }^{2}$ (D) \\ 1 Industrial Safety Department, Saint Petersburg Mining University, 21st Line 2, 199106 St. Petersburg, Russia \\ 2 The Strength Physics Laboratory, Ioffe Institute, 26 Polytekhnicheskaya, 194021 St. Petersburg, Russia; \\ h.machmoudov@mail.ioffe.ru \\ * Correspondence: afanasev_pi@pers.spmi.ru
}

Citation: Afanasev, P.I.; Makhmudov, K.F. Assessment of the Parameters of a Shock Wave on the Wall of an Explosion Cavity with the Refraction of a Detonation Wave of Emulsion Explosives. Appl. Sci. 2021, 11, 3976. https://doi.org/10.3390/app11093976

Academic Editor: Irina Bayuk

Received: 24 March 2021

Accepted: 27 April 2021

Published: 27 April 2021

Publisher's Note: MDPI stays neutra with regard to jurisdictional claims in published maps and institutional affiliations.

Copyright: (c) 2021 by the authors. Licensee MDPI, Basel, Switzerland. This article is an open access article distributed under the terms and conditions of the Creative Commons Attribution (CC BY) license (https:/ / creativecommons.org/licenses/by/ $4.0 /)$.

\begin{abstract}
At present, studying the parameters of shock waves at pressures up to $20 \mathrm{GPa}$ entails a number of practical difficulties. In order to describe the propagation of shock waves, their initial parameters on the wall of the explosion cavity need to be known. With the determination of initial parameters, pressures in the near zone of the explosion can be calculated, and the choice of explosives can be substantiated. Therefore, developing a method for estimating shock wave parameters on an explosion cavity wall during the refraction of a detonation wave is an important problem in blast mining. This article proposes a method based on the theory of breakdown of an arbitrary discontinuity (the Riemann problem) to determine the shock wave parameters on the wall of the explosion cavity. Two possible variants of detonation wave refraction on the explosion cavity wall are described. This manuscript compares the parameters on the explosion cavity wall when using emulsion explosives with those obtained using cheap granular ANFO explosives. The detonative decomposition of emulsion explosives is also considered, and an equation of state for gaseous explosion products is proposed, which enables the estimation of detonation parameters while accounting for the incompressible volume of molecules (covolume) at the Chapman-Jouguet point.
\end{abstract}

Keywords: analytic model; explosive; shock waves; refraction of a detonation wave; blast pressure

\section{Introduction}

Mining works currently use emulsion explosives, which are safe to manufacture, transport, store and employ for borehole charging [1-5], because they are devoid of explosive initiators such as trotyl, hexogen and other high-brisance explosives. The basic composition of emulsion explosives is comparable to that of cheap granular explosives (igdanites and granulites) [6], but emulsion explosives are multi-component fluids. The size of the particles of an emulsion explosive and their distribution in the dispersed medium are the main criteria for assessing the quality of the explosive preparation. Emulsion explosives are generally classified into two types: oil-in-water and water-in-oil. The emulsion composition of the oil-in-water type, according to its physical properties and chemical composition, is characteristic of slurry explosives, since they contain a structuring agent and thickener, and the combustible component of the emulsion matrix is fuel in a solution of water and an oxidizer. The stability of the properties of such an emulsion is based on the selection of the required emulsifier. Another component of the water-in-oil type is called emulite, which is an emulsion of a highly concentrated water solution of oxidizer salts (up to 80 percent of dissolved ammonium nitrate salts) in the fuel phase. Thus, water-in-oil emulsions have a higher resistance to water, as the smallest droplets of the oxidizing solution are inside a thin waterproof film of the fuel phase.

Two theories are used to describe detonating explosives: (1) The hydrodynamic theory of Zeldovich-von Neumann-Döring [7,8] and (2) the "hot spot" theory [9-13]. 
The hydrodynamic theory of Zeldovich-von Neumann-Döring describes the detonation of explosives initiated by shock waves. The chemical decomposition of emulsion explosives by detonation is dependent on the presence of spherical particles, which can be obtained through the use of plastic microspheres or gas-generating agents. A deficiency of spherical particles makes it impossible to initiate an emulsion explosive through the use of a booster explosive charge (use of a shock wave). Therefore, a number of researchers applied the "hot spot" theory as an alternative to describe the detonation of emulsion explosives. The "hot spot" theory, originally developed to describe the initiation of an explosion by impact or friction, is based on concepts involving the structure of matter, which varies from a continuous medium to a molecular structure. According to the theory of "hot spots", combustion mechanisms can be divided into the following: ignition on glide surfaces, ignition in the vicinity of collapsing spherical particles, ignition in the zones of adiabatic shear in crystal, ignition on the edge of spreading cracks and combustion of explosive particles penetrating the gas bubble $[10,14]$. All of these mechanisms are based on a thermal explosion, which occurs if the rate of heat generation by the chemical reaction exceeds the rate of heat rejection to the external environment. The self-heating of the emulsion occurs in accordance with the Arrhenius equation without branched chains in the chemical reaction.

The duration and amplitude of the action differ between shock wave initiation and impact initiation, and there is an absence of heat for shock wave initiation since it does not exchange heat with the environment. Therefore, the hydrodynamic theory is appropriate for describing the detonation of an emulsion explosive.

A large number of equations of state have been devised to describe the behavior of gaseous explosion products, and they can be divided into two main groups: (1) equations of state that include the chemical composition of gaseous explosion products (the BeckerNedostup equation, the Abel equation, the Berthelot equation, the Becker-KistiakowskyWilson equation); (2) equations of state that do not include the chemical composition of the explosion products but describe them as an average (the theta equation of state, Landau-Stanyukovich equation of state, Jones-Wilkins-Lee equation of state). In practice, the first group of equations of state are able to predict the detonation characteristics of, as a rule, gaseous explosive mixtures and, less often, condensed explosives. Equations of state in the second group can be used to solve problems applicable to mining.

The Jones-Wilkins-Lee equation of state, which is widely used in the scientific literature [15-20], has six empirical (adjustable) constants. On the one hand, this provides for a good description of the behavior of gaseous explosion products, but the resulting combinations of empirical constants are not precisely determinable, and it is always possible to choose another set of constants that will describe the behavior of gaseous explosion products in addition to the first set. This equation is effective for describing well-characterized high-brisance explosives (trotyl, hexogen, ten), but for industrial explosives, an equation of state that does not require an additional basis for its application is more practical. This approach is presented in this work and has been previously described [21].

The main premise of the applied equation of state (1) is as follows: in the zone of the chemical reaction, the condensed explosive is in a highly compressed state; the atoms or molecules of the explosive are in oscillatory motion, rather than thermal motion, and the repulsive forces determine the pressure magnitude. To describe such a system, methods of statistical physics for expressing Helmholtz energy are applied. Further gaseous explosion products appear at the Chapman-Jouguet point, contributing to the magnitude of the detonation pressure (namely, reducing it) because there is a volume of molecules that cannot be compressed. Then, it defines the pressure at the Chapman-Jouguet point, which is the initial pressure for the polytropic expansion of gaseous explosion products.

The next important objective of this research is to determine the parameters on the wall of an explosion cavity. At present, when designing blasting operations in open-pit mines, the shock wave parameters in the rock are not calculated. The main approach to determining these parameters for drilling and blasting operations and choosing the 
applicable explosive is based on the empirical ratio of the required amount of explosive to the volume of rock moved in space. This approach does not consider physical processes of transmitting the detonation pressure into the rock; therefore, it is necessary to study these physical processes to assess the expediency of using an explosive and to prepare the initial data for calculating the parameters of drilling and blasting operations based on the propagation of shock waves.

Many scientists use acoustic approximation to determine the pressure on the explosion cavity wall [21]:

$$
P_{f}=\frac{\rho_{m} \cdot C_{p}}{\rho_{m} \cdot C_{p}+\rho_{v v} \cdot D} P_{d}
$$

where $P_{d}$ is the pressure of detonation products at the Chapman-Jouguet point, $\rho_{m}$ is the density of the rock, $C_{p}$ is the velocity of a longitudinal wave, $\rho_{v v}$ is the density of the explosive, and $D$ is the velocity of detonation.

In the framework of the acoustic approach, it is initially assumed that the shock wave has a small amplitude that appears at a relatively large equilibrium pressure, and it is also assumed that the shock wave propagates with the local velocity of sound. Comparing the calculated pressure in the rock with experimental data reveals up to a twofold discrepancy.

The propagation of shock waves in solids at relatively low pressures causes two configurations of waves: the first to propagate is a wave called an elastic precursor, and the second is a shock wave (often called a plastic wave), which propagates with the volume velocity of sound (Figure 1).

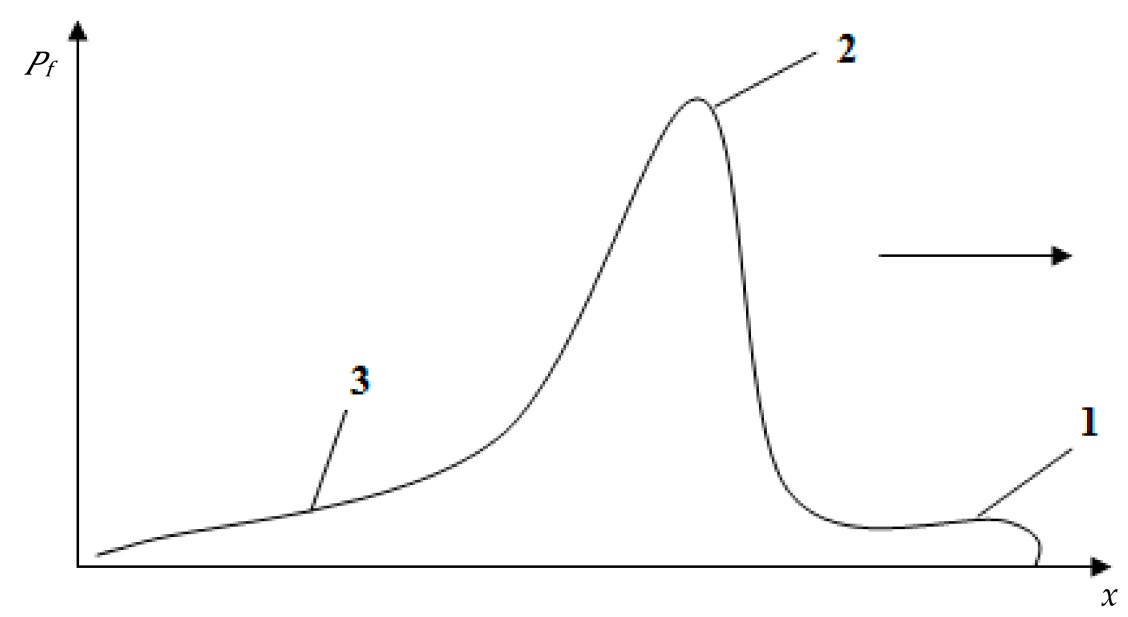

Figure 1. Schematic shape of the shock wave in the rock. 1-elastic precursor; 2-plastic wave; 3-rarefaction waves and diffracted waves.

The plastic wave velocity is determined by the formula [9]

$$
C_{V}=\sqrt{\frac{1+v}{3(1-v)}} C_{p},
$$

where $v$ is Poisson's ratio.

There is one other significant consideration: shock waves have no equilibrium pressure at all because they are characterized by a rapid increase and decrease in pressure in a short period of time (approximately $10^{-6}-10^{-5} \mathrm{~s}$ ); thus, the equilibrium state is unattainable.

Previously applied methods for estimating shock wave parameters on the borehole wall are based on the interaction of an ideal gas with the wall of the explosion cavity and the application of acoustic approximation to the refraction of a detonation wave. The values resulting from these approaches are underestimated in comparison with experimental results; therefore, it is necessary to account for the physical processes that occur during the refraction of detonation waves in the rock. 


\section{Materials and Methods}

The behavior of detonation products can be described with the equation of state in [10]:

$$
P_{d}=\left(1-\alpha_{k o v} \cdot \rho_{v v}\right) \frac{\rho_{v v} \cdot D^{2}}{(n+1)}
$$

where $n$ is a polytropic exponent, $\alpha_{k o v}=10^{-3} e^{-0,4 \cdot 10^{-3}} \cdot \rho_{d}$ is an incompressible volume of molecules [8], $D$ is the detonation velocity, $\rho_{v v}$ is the explosive density, and $\rho_{d}$ is the density of gaseous products of the explosion behind the chemical reaction zone.

The polytropic exponent is determined via the Landau-Stanyukovich equation [22]:

$$
D^{2}=\left[4 A \cdot(n+1)+2\left(n^{2}-1\right)\right] \cdot Q+10^{10} \cdot \rho_{v v}^{n-1} \cdot b
$$

where $A, b$ are empirical coefficients, and $Q$ is explosion heat.

The density of gaseous explosion products is determined with the formula [10]

$$
\rho_{d}=\rho_{v v} \frac{n+1}{\left(n+\alpha_{k o v} \cdot \rho_{v v}\right)} .
$$

The interaction of the detonation front with the surface of the blasthole wall should be determined using general approaches involving the theory of breakdown of an arbitrary discontinuity. The surface of an arbitrary discontinuity is considered to be part of the space where gas-dynamic and thermodynamic parameters of the medium change extremely rapidly. In order to calculate the shock wave values at the initial moment in the immediate vicinity of the borehole surface between the detonation products and the rock, one should use the general regularities of the shock wave that propagates in the detonation products and into the rock, which borders on the explosive charge. Another condition that determines the solution for the detonation wave refraction in the rock is the equality of the pressures and velocities of the particles on the surface of discontinuity, which is ensured by changing the flow parameters in the detonation products.

When the detonation wave is refracted on the explosion cavity wall in the rock, different wave refraction mechanisms for emulsion explosives are realized: (1) the pressure values of the detonation products can be higher than the shock wave; (2) the pressure values of the detonation products may be lower than the shock wave.

(1) If the pressure of the detonation products is higher than that of the shock wave, then a rarefaction wave propagates in the detonation products, and a shock wave propagates in the rock.

The velocity of particles in detonation products after refraction of the detonation wave is determined in accordance with the first variant:

$$
u_{f}=\Delta u+u_{d}
$$

where $u_{d}$ is the velocity of detonation product particles at the Chapman-Jouguet point, $\mathrm{m} / \mathrm{s} ; u_{f}$ is the velocity of the rock particles, $\mathrm{m} / \mathrm{s}$; and $\Delta u$ is the increase in the velocity of detonation product particles, $\mathrm{m} / \mathrm{s}$.

The increase in velocity can be calculated by determining the change in the velocity of the particles in the detonation products. The following integral was obtained by G. Riemann [9]:

$$
\Delta u=\int_{P_{f}}^{P_{d}} \frac{d p}{c \rho}
$$

where $\rho$ is the density of detonation products after refraction, and $c$ is the velocity of sound in the detonation products after refraction. 
The velocity of detonation product particles and the velocity of sound at the ChapmanJouguet point are obtained [9]:

$$
u_{d}=\frac{D}{n+1}, c_{d}=\frac{n D}{n+1}
$$

The velocity of sound in the detonation products is described by the formula

$$
\mathrm{c}=\sqrt{\partial p / \partial \rho},
$$

where $p=f(\rho)$ is the law of the polytropic expansion of detonation products.

After rearrangement, the incompressible volume of molecules is calculated:

$$
\begin{gathered}
c=\left(\frac{P}{P_{d}}\right)^{\frac{n-1}{2 n}} \frac{\left(1-\alpha_{k o v} \cdot \rho_{v v}\right) n D}{n+1} \\
\rho=\left(\frac{P}{P_{d}}\right)^{\frac{1}{n}} \frac{\rho_{d}}{\left(1-\alpha_{k o v} \cdot \rho_{d}\right)}
\end{gathered} .
$$

Then, Formula (7) is placed in the Riemann integral (5). The integration of a definite integral (5) is conducted according to the pressure range from the explosive to the pressure in the rock, and thus, we obtain the formula:

$$
\Delta u=\frac{2\left(n+\alpha_{k o v} \cdot \rho_{v v}\right)}{(n-1) \rho_{v v} \cdot D} P_{d}\left[1-\left(\frac{2 P_{f}}{P_{d}}\right)^{\frac{n-1}{2 n}}\right],
$$

where $P_{f}$ is the pressure in the front of the shock wave in the medium, $\mathrm{Pa} ; P_{d}$ is the pressure in the Chapman-Jouguet, Pa; and $D$ is explosive velocity, $\mathrm{m} / \mathrm{s}$.

Thus, after the breakdown of an arbitrary discontinuity, the total velocity of particles in the detonation products constitutes

$$
u_{d}+\Delta u=\frac{D}{n+1}+\frac{2\left(n+\alpha_{k o v} \cdot \rho_{v v}\right)}{(n-1) \rho_{v v} \cdot D} P_{d}\left[1-\left(\frac{2 P_{f}}{P_{d}}\right)^{\frac{n-1}{2 n}}\right] .
$$

(2) If the pressure of the detonation products is lower than that of the shock wave, then a secondary compression shock wave propagates in the detonation products, and a shock wave propagates in the rock [23]. On both sides of the boundary, the velocities of particles in the environment and in detonation products become equal:

$$
u_{f}=u_{d}-\Delta u .
$$

In this case, the decrease in the velocity of the particles is determined as follows:

$$
\Delta u=\sqrt{\left(P_{f}-P_{d}\right)\left(\frac{1}{\rho_{d}}-\frac{1}{\rho_{f}}\right)} .
$$

The Hugoniot equation for a refracted shock wave is

$$
\frac{\rho_{d}}{\rho_{f}}=\frac{(n+1) P_{d}+(n-1) P_{f}}{(n+1) P_{f}+(n-1) P_{d}}=\frac{(n-1) \pi+(n+1)}{(n+1) \pi+(n-1)}
$$

where $\pi=\frac{P_{f}}{P_{d}}$. 
Substituting the Hugoniot equation of the shock wave (12) into the dependence (11) results in

$$
\Delta u=\sqrt{P_{d} \frac{1}{\rho_{d}}(\pi-1)\left(1-\frac{(n-1) \pi+(n+1)}{(n+1) \pi+(n-1)}\right)}
$$

After substituting $P_{d}, \rho_{d}$ from $(1,3)$ and rearranging, we obtain

$$
\Delta u=\left(P_{f}-P_{d}\right) \sqrt{\left(\frac{2\left(n-\alpha_{k o v} \cdot \rho_{v v}\right)}{\rho_{v v}\left[(n+1) P_{f}+(n-1) P_{d}\right]}\right)} .
$$

The formula for determining the velocity of the particles in detonation products after the refraction of a detonation wave is

$$
u_{d}-\Delta u=\frac{D}{n+1}-\left(P_{f}-P_{d}\right) \sqrt{\left(\frac{2\left(n-\alpha_{k o v} \cdot \rho_{v v}\right)}{\rho_{v v}\left[(n+1) P_{f}+(n-1) P_{d}\right]}\right)},
$$

The shock wave in the rock can be presented in the following form [14]:

$$
P_{f}=\rho_{m}\left(C_{v}+\frac{G+1}{2} u_{f}\right) u_{f},
$$

where $u_{f}$ is the velocity of a rock particles, $C_{v}$ is the velocity of the plastic wave of compression, and $G$ is the Grüneisen parameter.

This type of shock wave was selected due to the physical nature of shock. The calculated values do not exceed $20 \mathrm{GPa}$ and are waves of low intensity; therefore, it is not necessary to account for the "thermal" component of the pressure. The calculated pressures are the "cold" component of the shock pressure, because the "thermal" component of the pressure increases the total pressure by no more than $8 \%$ of the shock pressure range of 1-20 GPa [9]. In addition, the rock is not highly porous, so there is no need to account for pore compressibility [24,25].

By equating the velocity of rock particles expressed in (16) to the velocity of particles in the detonation products $(9,15)$, we can use an iterative method to calculate the shock pressure at the wave front, followed by the velocity of the rock particles.

The solution of the above equations will determine the pressure on the wall of the explosion cavity:

$$
\begin{gathered}
\frac{2\left(n+\alpha_{k o v} \cdot \rho_{v v}\right)}{(n-1) \rho_{v v} \cdot D} P_{d}\left[1-\left(\frac{2 P_{f}}{P_{d}}\right)^{\frac{n-1}{2 n}}\right]+u_{d}-\left(\frac{C_{v}}{2}+\sqrt{\frac{\left(\rho_{m} C_{v}\right)^{2}+4 P_{f} \rho_{m}}{2 \rho_{m}}}\right)=0, \\
-\left(P_{f}-P_{d}\right) \sqrt{\left(\frac{2\left(n-\alpha_{k o v} \cdot \rho_{v v}\right)}{\rho_{v v}\left[(n+1) P_{f}+(n-1) P_{d}\right]}\right)}+u_{d}-\left(\frac{C_{v}}{2}+\sqrt{\frac{\left(\rho_{m} C_{v}\right)^{2}+4 P_{f} \rho_{m}}{2 \rho_{m}}}\right)=0 .
\end{gathered}
$$

The presented equations were solved in the MATLAB mathematical package. A block diagram of the solution is presented below (Figure 2). 

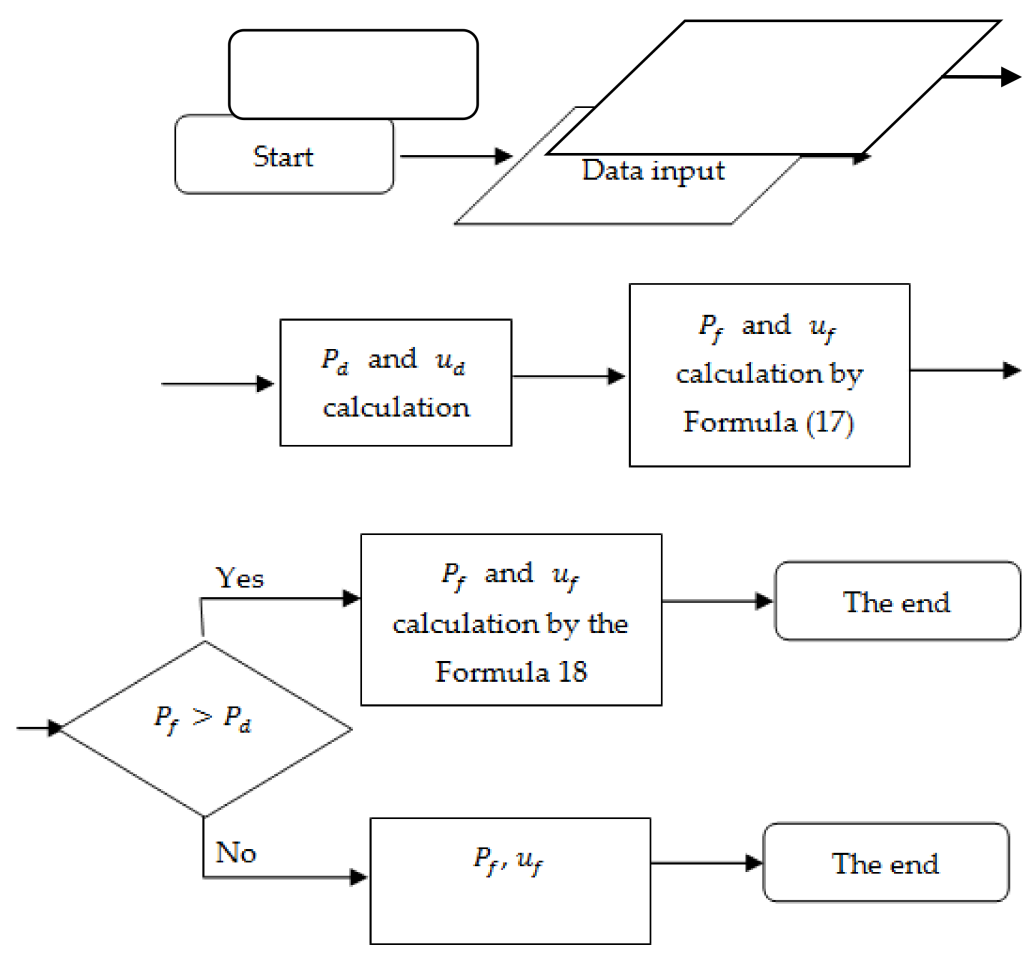

Figure 2. A block diagram of the solution

\section{Results and Discussion}

The calculated parameters of shock waves at the boundary of the explosion cavity were compared with experimental data from a previous study [21]. Figure 3 shows the experimental and calculated points. Experimental data were obtained for trotyl as an explosive (Table 1) in different types of rocks (diabase, granite, crystalline limestone, limestone) (Table 2).

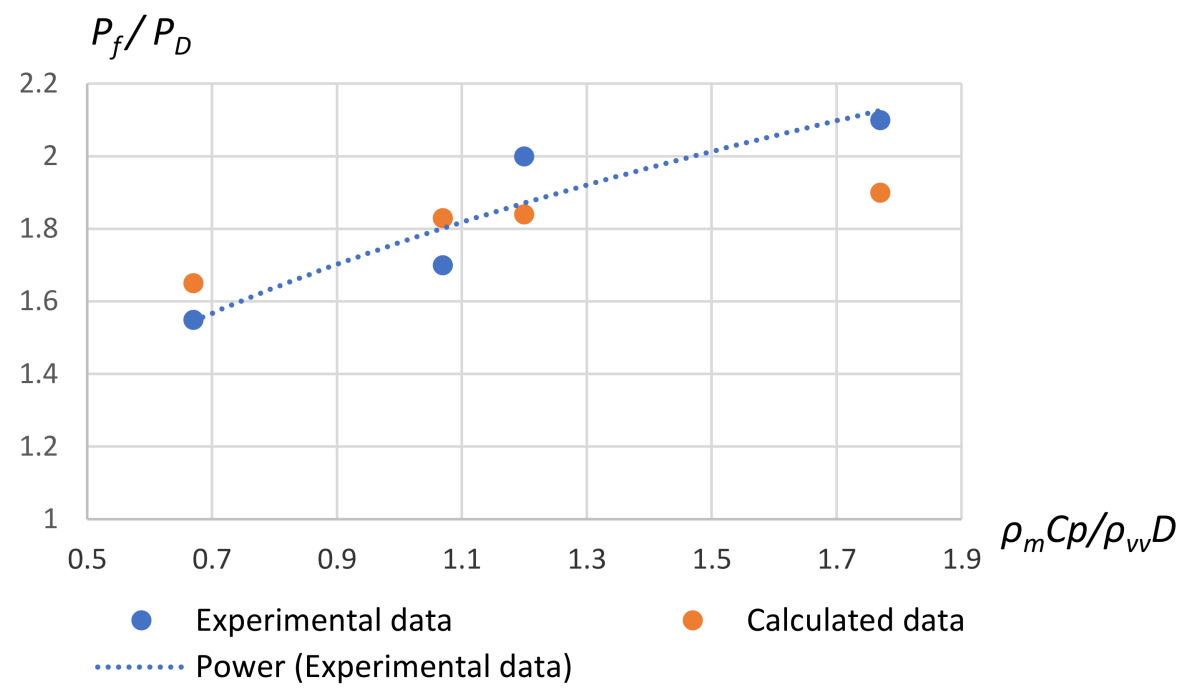

Figure 3. Comparison of experimental and calculated data. Dependence of the ratio of pressure transmission into rock on the impedances of the rock and explosive. 
Table 1. Detonation characteristics of trotyl.

\begin{tabular}{|c|c|c|c|c|c|c|}
\hline \multirow[b]{2}{*}{ Type of Explosive } & \multicolumn{3}{|c|}{ Main Characteristics } & \multirow[b]{2}{*}{$\gamma$} & \multirow{2}{*}{$\begin{array}{c}\mathbf{u}_{\mathrm{d}} \\
{[\mathrm{m} / \mathrm{s}]}\end{array}$} & \multirow[b]{2}{*}{$\begin{array}{c}\mathbf{P}_{\mathbf{d}} \\
{[\mathrm{GPa}]}\end{array}$} \\
\hline & $\begin{array}{c}\rho_{\mathrm{vv}} \\
{\left[\mathrm{kg} / \mathrm{m}^{3}\right]}\end{array}$ & $\underset{[\mathrm{kJ} / \mathrm{kg}]}{\mathrm{Q}}$ & $\begin{array}{c}\mathrm{D} \\
{[\mathrm{m} / \mathrm{s}]}\end{array}$ & & & \\
\hline Granulotole & 1600 & 4600 & 7000 & 3.19 & 1668 & 3.37 \\
\hline
\end{tabular}

Table 2. Physical-mechanical properties of the rocks.

\begin{tabular}{cccc}
\hline Rock & $\begin{array}{c}\boldsymbol{\rho}_{\mathbf{m}} \\
{\left[\mathbf{k g} / \mathbf{m}^{3}\right]}\end{array}$ & $\begin{array}{c}C_{p} \\
{[\mathbf{m} / \mathbf{s}]}\end{array}$ & Poisson Ratio \\
\hline Diabase & 3300 & 6000 & 0.15 \\
Granite & 2650 & 5100 & 0.2 \\
Crystalline limestone & 2800 & 4300 & 0.23 \\
Limestone & 2500 & 3000 & 0.25 \\
\hline
\end{tabular}

The ratio of pressure transmission is determined:

$$
K_{R}=\frac{P_{f}}{P_{d}} .
$$

The impedance of the rock is determined:

$$
I_{R}=\rho_{m} C_{p} .
$$

The impedance of the explosive is determined:

$$
I_{E}=\rho_{v v} \mathrm{D}
$$

Therefore, the experimental data are consistent with the calculated data (Figure 3). This method was used to calculate the refraction parameters of the detonation wave of emulsion explosives. The following explosives were selected: Nitronite E-70, Emulsolite A-20 and ANFO.

Nitronite E-70 is an emulsion explosive designed for blasting operations in open-cut mining fields for rocks with a strength coefficient of up to 20 on the Protodyakonov scale. It is a mechanical mixture of an emulsion, porous granular ammonium nitrate, diesel fuel and a gas-generating agent. An analog of Nitronite E-70 is Emulsolite A-20, but they differ in the content of the gas-generating agent: for Nitronite E-70, the gas-generating agent is one percent of the emulsion volume, and for Emulsolite A-20, it is one percent of the total volume. Moreover, of the two explosives, Emulsolite is more energetic due to its higher charging density and heat of explosion.

ANFO is a granular explosive and is a stoichiometric mixture of ammonium nitrate and diesel fuel. This explosive is not water-resistant, but it is cheaper to manufacture than emulsion explosives, so a granular explosive is always used in mining.

As mentioned earlier, since these explosives are initiated with the use of a booster explosive charge in the mining industry, the shock wave detonation mechanism is implemented; therefore, the equation of state (1) is used to determine the parameters of the detonation wave. With this type of initiation, the heat required to start the detonation according to the "hot spot" mechanism is absent, but the shock pressure in the explosive increases very rapidly, reaching the maximum value within 12 microseconds.

The main detonation characteristics of the analyzed explosives, which are presented in Table 3, were used as data for the computation. 
Table 3. Detonation characteristics of explosives.

\begin{tabular}{|c|c|c|c|c|c|c|}
\hline \multirow[b]{2}{*}{ Explosive } & \multicolumn{3}{|c|}{ Main Characteristics } & \multirow[b]{2}{*}{$\gamma$} & \multirow{2}{*}{$\begin{array}{c}\mathbf{u}_{\mathbf{d}} \\
{[\mathrm{m} / \mathrm{s}]}\end{array}$} & \multirow[b]{2}{*}{$\begin{array}{c}\mathbf{P}_{\mathrm{d}} \\
{[\mathrm{GPa}]}\end{array}$} \\
\hline & $\begin{array}{c}\rho_{\mathrm{vv}} \\
{\left[\mathrm{kg} / \mathrm{m}^{3}\right]}\end{array}$ & $\underset{[\mathrm{kJ} / \mathrm{kg}]}{\mathrm{Q}}$ & $\begin{array}{c}\mathrm{D} \\
{[\mathrm{m} / \mathrm{s}]}\end{array}$ & & & \\
\hline Nitronite E-70 & 1150 & 3200 & 5000 & 3.01 & 1270 & 2.15 \\
\hline Emulsolite A-20 & 1250 & 3500 & 4900 & 2.86 & 1268 & 2.1 \\
\hline ANFO & 900 & 3200 & 3700 & 2.66 & 1012 & 1.35 \\
\hline
\end{tabular}

The following physical-mechanical properties of rocks that were used for calculations $[25,26]$ (see Table 4).

Table 4. Physical-mechanical properties of the rocks.

\begin{tabular}{cccc}
\hline Rock & $\begin{array}{c}\boldsymbol{\rho}_{\mathbf{m}} \\
{\left[\mathbf{k g} / \mathbf{m}^{3}\right]}\end{array}$ & $\begin{array}{c}\mathbf{C}_{\mathbf{p}} \\
{[\mathbf{m} / \mathbf{s}]}\end{array}$ & Poisson's Ratio \\
\hline Granite & 2600 & 5000 & 0.23 \\
Sandstone & 2200 & 2100 & 0.16 \\
Siltstone & 2200 & 1400 & 0.28 \\
\hline
\end{tabular}

After substituting the initial data (Tables 3 and 4) into Formulas (17) and (18), the parameters of the refraction detonation wave on the borehole wall (Table 5) can be calculated.

Table 5. The pressure on the wall of the explosion cavity in rocks.

\begin{tabular}{cccc}
\hline \multirow{2}{*}{ Explosive } & Granite & Sandstone & Siltstone \\
\cline { 2 - 4 } & & $\mathbf{P}_{\mathbf{f}}[\mathrm{GPa}]$ & \\
\hline Nitronite E-70 & 3.69 & 3.08 & 2.96 \\
Emulsolite A-20 & 3.72 & 3.08 & 2.96 \\
ANFO & 2.29 & 1.97 & 1.88 \\
\hline
\end{tabular}

As a result, there is a change in the ratio of pressure transmission to different rocks for a given explosive and an increase in the acoustic impedance of the rock. In addition, the pressure transmission ratios for different explosives in the same rock do not significantly differ (Table 6).

Table 6. Ratio of pressure transmission into rocks.

\begin{tabular}{cccc}
\hline \multirow{2}{*}{ Rock } & \multicolumn{3}{c}{ Ratio of Pressure Transmission } \\
\cline { 2 - 4 } & Nitronite E-70 & Emulsolite A-20 & ANFO \\
\hline Granite & 1.71 & 1.77 & 1.7 \\
Sandstone & 1.43 & 1.46 & 1.46 \\
Siltstone & 1.37 & 1.41 & 1.42 \\
\hline
\end{tabular}

The obtained results (Table 6) reveal that, despite differences in the detonation parameters of explosives, the shock wave receives a certain amount of the detonation pressure. This is due to the redistribution of motion in the gaseous explosion products because a wave of secondary compression arises in them.

\section{Conclusions}

The following conclusions can be drawn from the assessment in this study.

1. The method described for calculating shock wave parameters on the explosion cavity wall accounts for physical processes that occur during the refraction of a detonation wave, which enables the analysis of the blasting effect on the rock. 
2. Despite the difference in the detonation parameters of explosives, only some of the detonation pressure is transmitted to the rock. The ratio of pressure transmission largely depends on the properties of the rock.

3. For acoustically rigid rocks, more brisant explosives must be used to obtain the highest ratio of pressure transmission. For acoustically soft rocks, less brisant explosives should be used.

4. The calculation of the ratio of pressure transmission for a specific rock will allow mining blasting engineers to adjust the characteristics of the explosive to reduce rock over-crushing and, as a result, lead to the rational use of explosion energy.

Author Contributions: Conceptualization, P.I.A. and K.F.M.; methodology, P.I.A. and K.F.M.; software, P.I.A.; validation, K.F.M.; formal analysis, K.F.M.; investigation, P.I.A. and K.F.M.; resources, P.I.A.; writing-original draft preparation, P.I.A.; writing-review and editing, K.F.M.; funding acquisition, P.I.A. All authors have read and agreed to the published version of the manuscript.

Funding: This work was supported by the Saint Petersburg Mining University under contract no. KPD1-20-178).

Institutional Review Board Statement: Not applicable.

Informed Consent Statement: Not applicable.

Data Availability Statement: Not applicable.

Conflicts of Interest: The authors declare no conflict of interest.

\section{References}

1. Shabarov, A.N.; Tsirel, S.V. Geodynamic safety in underground mining. Gorn. Zhurnal 2017, 9, 65-70. [CrossRef]

2. Sidorenko, A.A.; Ivanov, V.V.; Dmitriyev, P.N. A study of gas drainage methods efficiency in Kotinskaya mine in Russia. ARPN J. Eng. Appl. Sci. 2020, 4, 530-535.

3. Sokolov, I.V.; Smirnov, A.A.; Rozhkov, A.A. Explosive blasting technology for strong valuable ores with fan-shaped wells. Zap. Gorn. Inst. 2019, 237, 285-291. [CrossRef]

4. Zuev, B.Y.; Zubov, V.P.; Fedorov, A.S. Application prospects for models of equivalent materials in studies of geomechanical processes in underground mining of solid minerals. Eurasian Min. 2019, 1, 8-12. [CrossRef]

5. Trushko, V.L.; Protosenya, A.G. Prospects for the development of geomechanics in a new technological paradigm. Zap. Gorn. Inst. 2019, 236, 162-166. [CrossRef]

6. Belin, V.A.; Paramonov, G.P.; Zhimyan, Z.H. Features of the manufacture and use of mixed explosives of the ASDT type at mining enterprises in Mongolia. Zap. Gorn. Inst. 2018, 232, 364-367. [CrossRef]

7. Baum, F.A.; Stanyukovich, K.P.; Shekhter, B.I. Explosion Physics; Nauka: Moscow, Russia, 1959.

8. Cook, M.A. Science of High Explosives; Nedra: Moscow, Russia, 1980.

9. Andreev, S.G.; Andreev, S.G.; Babkin, A.V. Explosion Physics; Fizmatlit: Moscow, Russia, 2004; ISBN 5-9221-0220-6.

10. Pavel, A.; Sergey, K.; Valentin, I. The equation of state for explosive detonation products. Int. J. Mech. Eng. Technol. 2018, 9, 865-868.

11. Bouden, F.; Ioffe, A. Excitation and Development of Explosion in Solids and Liquids; Ilim: Moscow, Russia, 1955.

12. Esen, S. A non-ideal detonation model for evaluating the performance of explosives in rock blasting. Rock Mech. Rock Eng. 2008, 41, 467-497. [CrossRef]

13. Cheng, Y.; Song, Z.; Jin, J.; Wang, J.; Yang, T. An experimental study on stress wave propagation and attenuation of sandstone during stress unloading process. J. Vib. Shock 2020, 39, 151-158.

14. Menzhulin, M.G.; Brovin, B.E. Formation of longitudinal and volumetric waves approximately the cavity during the explosion of explosives in rocks. Zap. Gorn. Inst. 2019, 180, 165-168.

15. Bourasseau, E.; Dubois, V.; Desbiens, N.; Maillet, J. Molecular simulations of Hugoniots of detonation products mixtures at chemical equilibrium: microscopic calculation of the Chapman-Jouguet state. J. Chem. Phys. 2007, 127, 084513. [CrossRef] [PubMed]

16. Couceiro, P. Modeling non-ideal velocity of detonation in rock blasting. REM Int. Eng. J. 2020, 73, 371-378. [CrossRef]

17. Minchinton, A. On the influence of fundamental detonics on blasting practice. In Proceedings of the 11th International Symposium on Rock Fragmentation by Blasting, Sydney, NSW, Australia, 24-26 August 2015; pp. 41-54.

18. Li, Y.; Cao, J.; Chen, X.; Huang, C.; Zhao, Q. Numerical investigation on crack formation and penetration mechanism between adjacent blast holes. Shock Vib. 2020, 2020, 8816059. [CrossRef]

19. Chi, L.Y.; Zhang, Z.-X.; Aalberg, A.; Li, C.C. Experimental investigation of blast-induced fractures in rock cylinders. Rock Mech. Rock Eng. 2019, 52, 2569-2584. [CrossRef]

20. Yi, C.; Johansson, D.U.; Nyberg; Beyglou, A. Stress wave interaction between two adjacent blast holes. Rock Mech. Rock Eng. 2016, 49, 1803-1812. [CrossRef] 
21. Borovikov, V.A.; Menzhulin, M.G. Stress Waves in a Watered Fractured Rock; Leningradskij Gornyj Institute: Leningrad, Russia, 1989.

22. Stanyukovich, K.P. Unsteady Motion of a Continuous Medium; Fizmatlit: Moscow, Russia, 1971.

23. Mitrofanov, V.V. Detonation of Homogenous and Heterogeneous Systems; Lavrentyev Institute of Hydrodynamics: Novosibirsk, Russia, 2003; ISBN 5-94571-003-6.

24. Pavlovich, A.A.; Korshunov, V.A.; Bazhukov, A.A.; Melnikov, N.Y.A. Assessment of the strength of the rock mass in the development of deposits by the open method. Zap. Gorn. Inst. 2019, 239, 502-509. [CrossRef]

25. Tsirel, S.V.; Pavlovich, A.A.; Melnikov, N.Y.; Zuev, B.Y. Physical Modeling of Deformation Processes in Pit Slope with Steep Bedding. J. Min. Sci. 2019, 55, 364-370. [CrossRef]

26. Zhabin, A.B.; Polyakov, A.V.; Averin, E.A.; Linnik, Y.U.N.; Linnik, V.Y.U. Assessment of the influence of abrasiveness of rocks on the parameters of rock cutting machines. Zap. Gorn. Inst. 2019, 240, 621-627. [CrossRef] 